\title{
La asincronía ventricular izquierda como resultado de la estimulación apical permanente por dispositivos en el ventrículo derecho
}

\section{Left ventricular dyssynchrony as result of right ventricular permanent apical pacing}

\author{
Diego X. Chango-Azanza1*, Martín A. Munín ${ }^{1}$, Gustavo A. Sánchez¹, Luis M. Arévalo-Pérez², \\ Juan J. Chango-Azanza ${ }^{3}$, María E. Pelayo ${ }^{2}$, Analía Paolucci4, Hugo Garro y Daniel Ortega ${ }^{4,5}$
}

'Servicio de Ultrasonido Cardiovascular del Centro de Educación Médica e Investigaciones Clínicas "Norberto Quirno" (CEMIC), Buenos Aires; ${ }^{2}$ Servicio de Electrofisiología Cardíaca del Hospital General de Agudos "Ramos Mejía", Buenos Aires; '3ervicio de Medicina Interna del Hospital Universitario de Connecticut, Farmington, EE.UU.; ${ }^{4}$ Servicio de Electrofisiología Cardíaca de la Clínica San Camilo, Buenos Aires; ${ }^{5}$ Fundación de Investigaciones de Bioingeniería Aplicada, Buenos Aires, Argentina

\begin{abstract}
Resumen
La estimulación apical permanente del ventrículo derecho (VD) puede producir asincronía del ventrículo izquierdo (VI) desde los puntos de vista eléctrico y mecánico. Este fenómeno es efecto de una alteración de la activación normal del VI que lleva al deterioro de la función sistólica y la aparición de insuficiencia cardíaca y sus efectos deletéreos relacionados. Para el estudio de la asincronía eléctrica del VI se ha propuesto en fecha reciente el nuevo sistema electrocardiográfico no invasivo Synchromax, que puede cuantificar el grado de asincronía eléctrica que causa una subsecuente asincronía mecánica. Esta última se ha estudiado casi siempre mediante la ecocardiografía transtorácica bidimensional (ETT2D) a través del Doppler tisular y la deformación miocárdica y ahora con la ecocardiografía tridimensional transtorácica en tiempo real (E3DTR). La relación entre estos fenómenos ha sido motivo de estudio a fin de identificar a los pacientes que se benefician de la transición a un tratamiento de resincronización cardíaca. Conclusiones: La estimulación artificial permanente del VD produce asincronía eléctrica del VI que puede cuantificarse mediante el nuevo sistema electrocardiográfico Synchromax y desencadenar asincronía mecánica estudiada mediante la ecocardiografía transtorácica para reconocer a los pacientes que pueden beneficiarse de un tratamiento de resincronización cardíaca.
\end{abstract}

Palabras clave: Asincronía ventricular izquierda. Estimulación apical permanente. Tratamiento de resincronización cardíaca.

\begin{abstract}
Permanent apical pacing of right ventricle (RV) can produce dyssynchrony of the left ventricle (LV) from an electrical and mechanical point of view. This phenomenon is caused by an alteration in the normal activation of $L V$ leading to a deterioration of systolic function and the appearance of heart failure and its associated deleterious effects. For the study of the electrical asynchrony of the LV, a new noninvasive electrocardiographic system Synchromax has recently been proposed, being able
\end{abstract}

\section{Correspondencia:}

*Diego X. Chango-Azanza

E-mail: diegochangomd@gmail.com
Disponible en internet: 14-09-2020 Arch Cardiol Mex. 2020;90(3):328-335

www.archivoscardiologia.com 1405-9940/๑ 2020 Instituto Nacional de Cardiología Ignacio Chávez. Publicado por Permanyer. Este es un artículo open access bajo la licencia CC BY-NC-ND (http://creativecommons.org/licenses/by-nc-nd/4.0/). 
to quantify the degree of electrical asynchrony that leads to a subsequent mechanical dyssynchrony. The latter has been traditionally studied by two-dimensional transthoracic echocardiography (2DTTE) through tissue Doppler and myocardial deformation and lately by real-time 3-dimensional echocardiography (RT3DE). The relationship between these phenomena has been the subject of study to predict those patients who benefit from an "upgrade" to cardiac resynchronization therapy. Conclusions: Permanent apical pacing of the RV produces electrical dyssynchrony of the $L V$ that can be quantified using a new electrocardiographic system Synchromax and trigger mechanical asynchrony studied through transthoracic echocardiography allowing to predict those patients who benefit from cardiac resynchronization therapy.

Key words: Left ventricular dyssynchrony. Permanent apical pacing. Cardiac resynchronization therapy.

\section{Introducción}

La estimulación permanente mediante dispositivos puede inducir disincronía eléctrica y mecánica al hacer que el ventrículo derecho (VD) se contraiga antes que el izquierdo (VI) (disincronía interventricular) y que el tabique interventricular lo haga antes que las paredes laterales (disincronía intraventricular) ${ }^{1}$. En términos históricos, se han descrito ya episodios clínicos adversos atribuidos a la estimulación del VD en pacientes con disfunción del nódulo sinusal, como tasas altas de insuficiencia cardíaca (IC) congestiva, fibrilación auricular, dilatación del VI y un incremento de la mortalidad².

La estimulación apical del VD ocasiona una alteración del patrón de activación del VI, similar al bloqueo de rama izquierda, y sobreviene una conducción eléctrica que inicia en el VD, activa al tabique interventricular de forma anómala (de derecha a izquierda) y da lugar al final a un notorio retraso de la activación de la pared lateral del $\mathrm{VI}$, con la consiguiente prolongación de la duración del $\mathrm{QRS}^{3}$, lo que provoca una contracción ventricular ineficiente con asincronía y pérdida del trabajo miocárdico ${ }^{4}$.

Tayal, et al. describieron un serie de 27,704 pacientes con un incremento significativo del riesgo de $10 \%$ para el desarrollo de IC en el transcurso de dos años luego del implante, una cifra similar a la de estudios previos². Además, existió un notorio mayor riesgo entre el primer y el sexto meses de seguimiento; los pacientes de sexo masculino, el infarto de miocardio previo y la insuficiencia renal crónica se relacionaron con mayor riesgo de desarrollar IC.

Otros estudios han mostrado un deterioro de la función sistólica determinado por la caída de la FEVI en promedio de $10 \%$ en los primeros días tras la implantación del dispositivo ${ }^{5}$. Por esta razón se considera que es necesario un seguimiento clínico y con ecocardiografía de estos pacientes, en especial en los primeros seis meses, lapso en el cual es probable identificar a la gran mayoría de sujetos con IC después del implante de un catéter ${ }^{6}$. Aquéllos con antecedente de infarto de miocardio tuvieron mayor disminución de la $\mathrm{FEVI}^{7}$, algo tal vez atribuido a la fibrosis miocárdica subyacente, mayor asincronía ventricular y una mayor anchura del QRS, de tal modo que el VI es más vulnerable al efecto perjudicial de la estimulación artificial ${ }^{8}$. Algo semejante se ha demostrado en portadores de enfermedad renal crónica, en la cual el mecanismo puede ser efecto de una cantidad considerable de fibrosis miocárdica ${ }^{9}$.

El tratamiento de resincronización cardíaca (TRC) ha demostrado beneficio en los pacientes que tienen indicación de estimulación permanente con IC y deterioro grave de la función sistólica $(\mathrm{FEVI}<35 \%)^{10}$. Las guías recientes de la European Society of Cardiology han incorporado esta premisa ${ }^{11}$; sin embargo, esto no es igual para pacientes con FEVI normal, en quienes existe evidencia limitada ${ }^{12}$, aunque podría plantearse esta conducta en enfermos con mayor riesgo de desarrollar IC, por ejemplo los individuos con antecedente de infarto agudo de miocardio y enfermedad renal crónica ${ }^{6}$.

Se ha observado un deterioro de la FEVI de $12.3 \%$ en una cohorte de pacientes que recibieron un marcapasos definitivo con previa función sistólica conservada del VI durante el seguimiento a $4.3 \pm 3.9$ años ${ }^{13}$, definido como una reducción menor del $40 \%$ o una disminución mayor del $10 \%$ de la FEVI previa. En un análisis multivariado de esta cohorte, un PEVD $\geq 20 \%$ se relacionó en sumo grado con deterioro de la FEVI. Se estableció además que aquéllos con hipertrofia ventricular izquierda y una tasa de estimulación mayor de $40 \%$ durante los últimos seis meses fueron parámetros relacionados con la presencia de mayor asincronía mecánica del $\mathrm{VI}^{14}$.

Como se ha mencionado, la FEVI es determinante para la indicación de TRC. Aunque el método biplano es el más robusto para determinar los volúmenes y la fracción de expulsión mediante la ETT2D, su variabilidad interobservador e intraobservador puede ser alta, con diferencias notificadas hasta de $18 \%{ }^{15}$. Los volúmenes ventriculares por esta técnica se han subestimado en comparación con la norma de referencia, esto es, la resonancia magnética cardíaca ${ }^{16}$. Cuando 


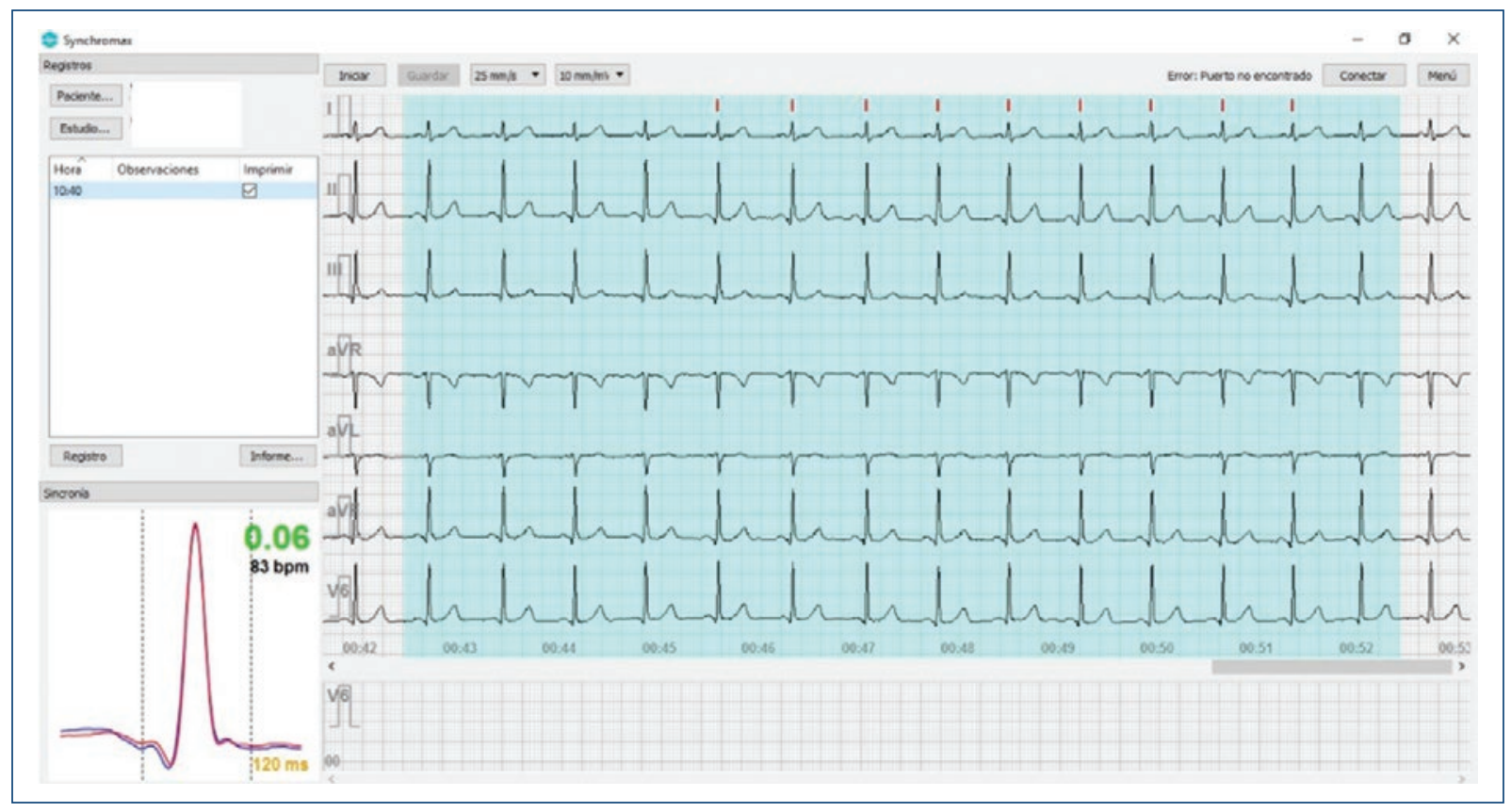

Figura 1. Secuencia de ritmo sinusal procesada por Synchromax. Procesamiento de la señal comparada de la derivación DII con la V6, con índice de disincronía normal (0.06).

se realizó la cuantificación de la FEVI respecto de la ETT2D y la resonancia magnética cardíaca se reconocieron discrepancias en cuanto a la indicación de TRC en $28 \%$ de los pacientes usando el punto de corte de la guía actual. La ecocardiografía con contraste puede reducir la variabilidad intraobservador e interobservador para el cálculo de los volúmenes y la fracción de expulsión ${ }^{17}$. Con base en estos hallazgos, el punto de corte para la selección de pacientes que se benefician de TRC depende de la herramienta de imagen utilizada, sin poder extrapolar un solo punto de corte para todas las herramientas de cálculo. Pese a ello, algún subgrupo de pacientes con fracción de expulsión de 30 a $40 \%$ podría incorporarse en las guías futuras ${ }^{18}$. Los individuos con FEVI mayor del punto de corte habitual, pero con un verdadero bloqueo completo de la rama izquierda, podrían beneficiarse de una TRC ${ }^{19}$.

\section{Synchromax para el estudio de la asincronía eléctrica}

La derivación DII del ECG de superficie sintetiza la actividad septal, dirección y velocidad a través de él, mientras que la derivación $\mathrm{V} 6$ registra la activación de la pared libre del VI. Por esa razón, en ausencia de trastornos de la conducción, la Dll es positiva y con una duración normal, una activación desde la base del SIV hasta la punta con velocidad normal y, por otro lado, V6 es casi idéntica, positiva y con igual duración y volumen espacial porque sigue el mismo patrón de activación. Mediante un cálculo matemático, el equipo toma las diferentes variables: dirección del impulso (de la base al vértice o viceversa), duración del QRS, volumen de ambas curvas, tiempo de máxima activación de cada cavidad y demoras en la propagación parietal, y luego realiza un índice de "sincronía" de 00 a 1.0 (00 es la sincronía perfecta y 1.00 la disincronía completa ${ }^{20}$ ).

A simple vista es posible observar algunos detalles imposibles de analizar en el ECG de superficie, entre ellos el sentido de la activación, de la base a la punta o viceversa (QRS positivo o negativo, respectivamente), la simultaneidad en la activación, y el retraso de la propagación (QRS ancho). Por ejemplo, en ritmo sinusal con QRS normal ambas curvas son simétricas, simultáneas angostas y con el mismo volumen (Fig. 1).

La anchura del QRS por sí sola no parece ser un marcador adecuado para diagnosticar disincronía ${ }^{21} \mathrm{y}$, si se considera la evidencia de que la dispersión morfológica de la despolarización ventricular es un 


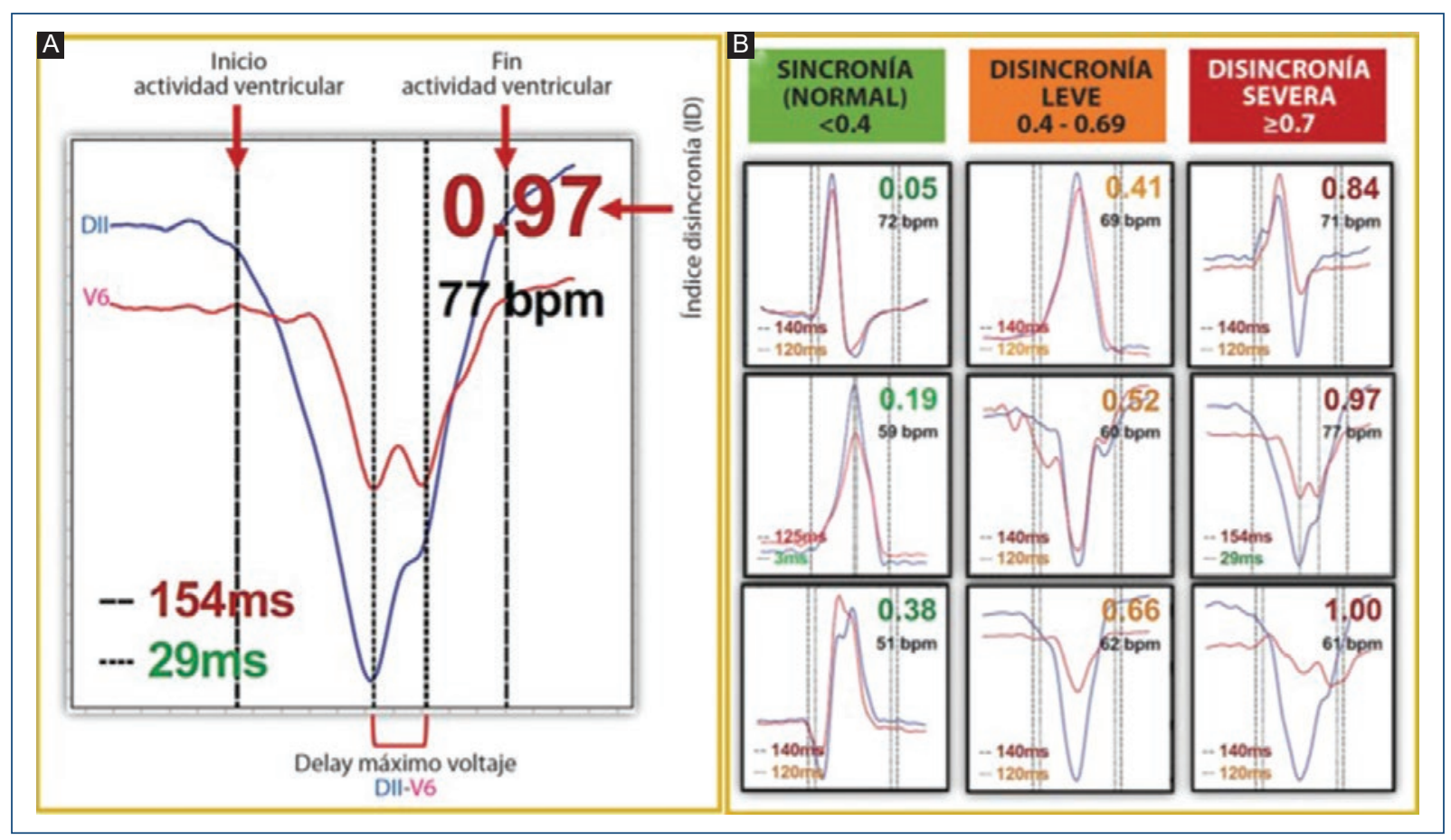

Figura 2. A: Comparación de las derivaciones DIl y V6 tomadas con Synchromax en un paciente con ritmo estimulado por marcapaso en el ventrículo derecho, tipo implantado apical. La activación del ventrículo derecho (VD) antecede a la del ventrículo izquierdo (VI) y el retardo del VI para máximo voltaje es de 29 milisegundos (ms); toda la actividad eléctrica ventricular toma 154 ms. B: Valores de corte para disincronía (trazos de Synchromax). En números verdes, de arriba abajo, dos casos de ECG normal y, al final, un caso de bloqueo de rama derecha (0.38). Al centro, en números naranjas, pacientes con bloqueo de rama izquierda $(0.41)$ y hemibloqueo anterior izquierdo $(0.52,0.66)$. A la derecha, con números rojos, de arriba abajo, pacientes con hemibloqueo anterior izquierdo (0.84), marcapaso en la punta del ventrículo derecho (0.97) y bloqueo avanzado de rama izquierda $(1.0)^{22}$.

marcador mucho más eficiente (como lo demuestran Bonomini, et al. con el concepto de varianza espacial del QRS 22,23 ), Synchromax es una herramienta óptima para estos fines, dado que de modo no invasivo e instantáneo, y sin procedimientos dependientes del operador, puede diagnosticar con criterios morfológicos (y no sólo espaciales) la disincronía eléctrica, además de que cuantifica la importancia de la disincronía eléctrica, tanto en el momento del implante como durante el seguimiento.

La estimulación desde el vértice del ventrículo derecho semeja un bloqueo de rama izquierda del haz de His: se pierden los vectores iniciales septales y el QRS se torna ancho y mellado; el eje eléctrico se desvía hacia la izquierda; la pared lateral se activa tardíamente y se produce disincronía electromecánica (Fig. 2) ${ }^{24}$ con la consecuente caída de la FEVI y la posible aparición de insuficiencia mitral, que reduce aún más la fracción de expulsión anterógrada ${ }^{19}$.
Cuando se trata de pacientes con dispositivos permanentes, que se encuentran crónicamente estimulados, y que estudió el sistema Synchromax, se ha encontrado que cerca del $72 \%$ de los enfermos alteró su asincronía eléctrica y un $54 \%$ mostró notable asincronía del VI (ID > 0.7) $)^{24}$.

\section{Estudio de la asincronía mecánica del VI mediante ecocardiografía}

La ecocardiografía y en particular las técnicas de Doppler tisular codificado en color son los métodos utilizados de forma más amplia para este fin, ya que poseen una alta resolución temporal y permiten el análisis de la motilidad segmentaria de una manera sencilla. No obstante, la asincronía mecánica ventricular analizada mediante Doppler tisular se basa en la motilidad ventricular longitudinal en una dimensión única y tiene como limitación principal la 


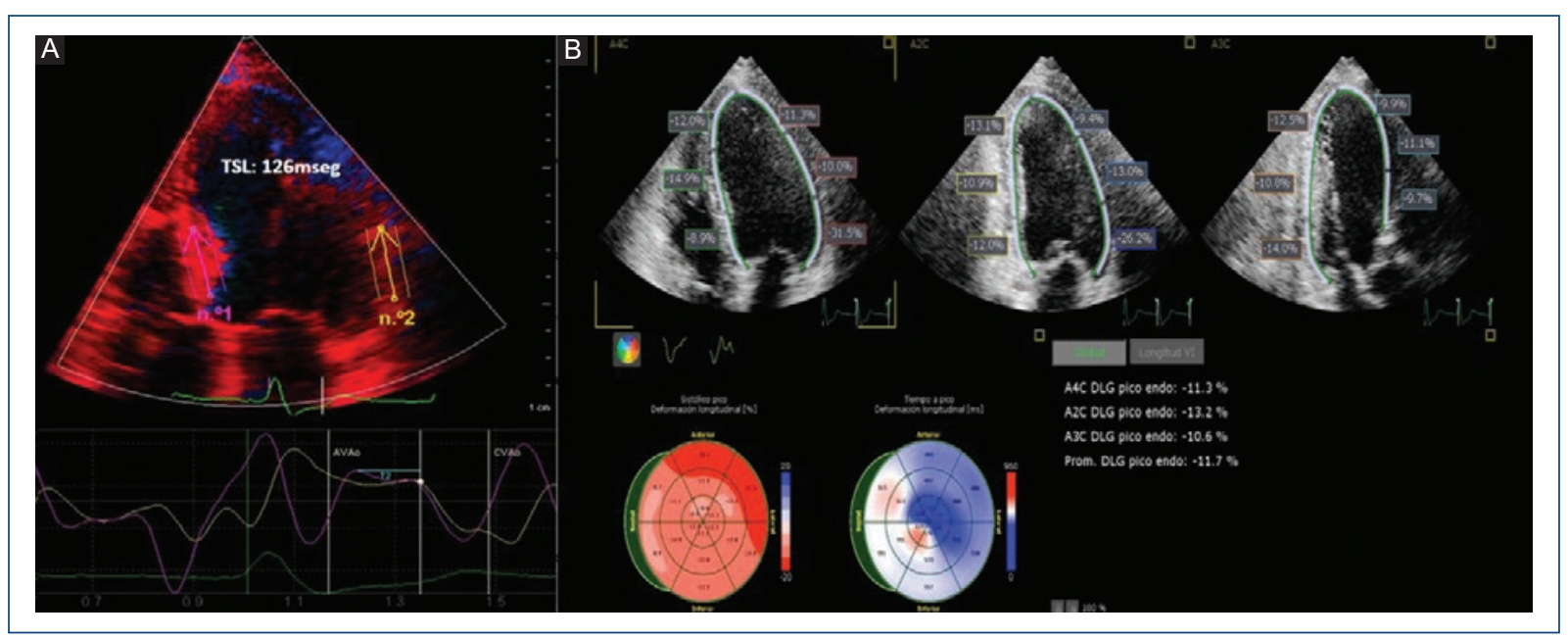

Figura 3. Análisis por ecocardiografía de la asincronía mecánica del VI en paciente con marcapasos permanente. A: Diferencia en tiempo septal-lateral (TSL) obtenida por Doppler tisular de 126 ms; se muestran n1 (línea púrpura) con activación septal y n2 (línea amarilla) con activación lateral del VI. B: Imágenes de deformación miocárdica longitudinal del VI obtenidas desde las vistas de cuatro, dos y tres cámaras, respectivamente. C: Esquema “ojo de buey" sobre deformación miocárdica longitudinal y tiempo al punto máximo sistólico de activación de todos los segmentos; se observan en azul las zonas con mayor retraso.

dependencia del ángulo de incidencia del haz de ultrasonido (Fig. 3A) ${ }^{25}$.

Las imágenes de deformación (strain) o el análisis de deformación con ecocardiografía emplean imágenes Doppler de tejido o ecocardiografía de seguimiento de "pecas" (Fig. 3B). Este último método es menos dependiente del ángulo y cubre toda la pared ventricular, en contraste con la imagen Doppler tisular, y por tanto es más confiable para la detección de la activación de segmentos retrasados (Fig. 3C). El retraso de la pared septal y lateral se obtiene con imágenes de velocidad Doppler tisular, calculada por la diferencia de tiempo entre el punto máximo de velocidad (Doppler) o la contracción (Doppler o seguimiento de "pecas") de la pared basal septal y lateral19.

\section{Valor agregado de la ecocardiografía tridimensional}

En fecha reciente, la ecocardiografía tridimensional transtorácica (E3DTR) en tiempo real ha demostrado que puede proveer el análisis del VI mediante una única adquisición de forma completa y simultánea. La evaluación simultánea del cambio sistólico de múltiples regiones de un volumen obtenido del VI y el cálculo del índice de asincronía sistólica (IAS) permitirían integrar información de una forma más fidedigna en comparación con otros métodos convencionales de la ecocardiografía bidimensional ${ }^{26}$.

Macron, et al. ${ }^{27}$ confirmaron que la E3DTR es una técnica simple, reproducible y de rápida evaluación, y hace posible además determinar los volúmenes ventriculares en telediástole y telesístole, el volumen sistólico y la fracción de expulsión del VI, además de cuantificar la asincronía mecánica ventricular respecto de la resonancia cardíaca ${ }^{28,29}$. En una serie de 35 pacientes con estimulación apical permanente se observó una disminución de $4.1 \%$ de la fracción de expulsión volumétrica, además de un significativo retraso de la activación de las paredes septal y posterior a nivel medioventricular, y reveló un IAS de $7 \pm 4 \%$ vs. $5.3 \pm 2.1 \%$ en individuos sin estimulación apical ${ }^{30}$.

La serie de un estudio de 93 pacientes que recibieron estimulación permanente del VD evaluados por ecocardiografía tridimensional mostró que el $49 \%$ de éstos tuvo parámetros de asincronía por E3DTR, toda vez que se compararon con 93 controles normales, en los que el valor de referencia a partir del cual existió asincronía mecánica ventricular se determinó como una desviación estándar del tiempo al mínimo volumen regional de los 16 segmentos ventriculares (Tmvr-16S) mayor que $16 \mathrm{~ms}$, correspondiente al tiempo que difiere de los controles normales en más de 2 desviaciones estándar. Estos pacientes con asincronía mecánica ventricular izquierda determinada a través de esta 


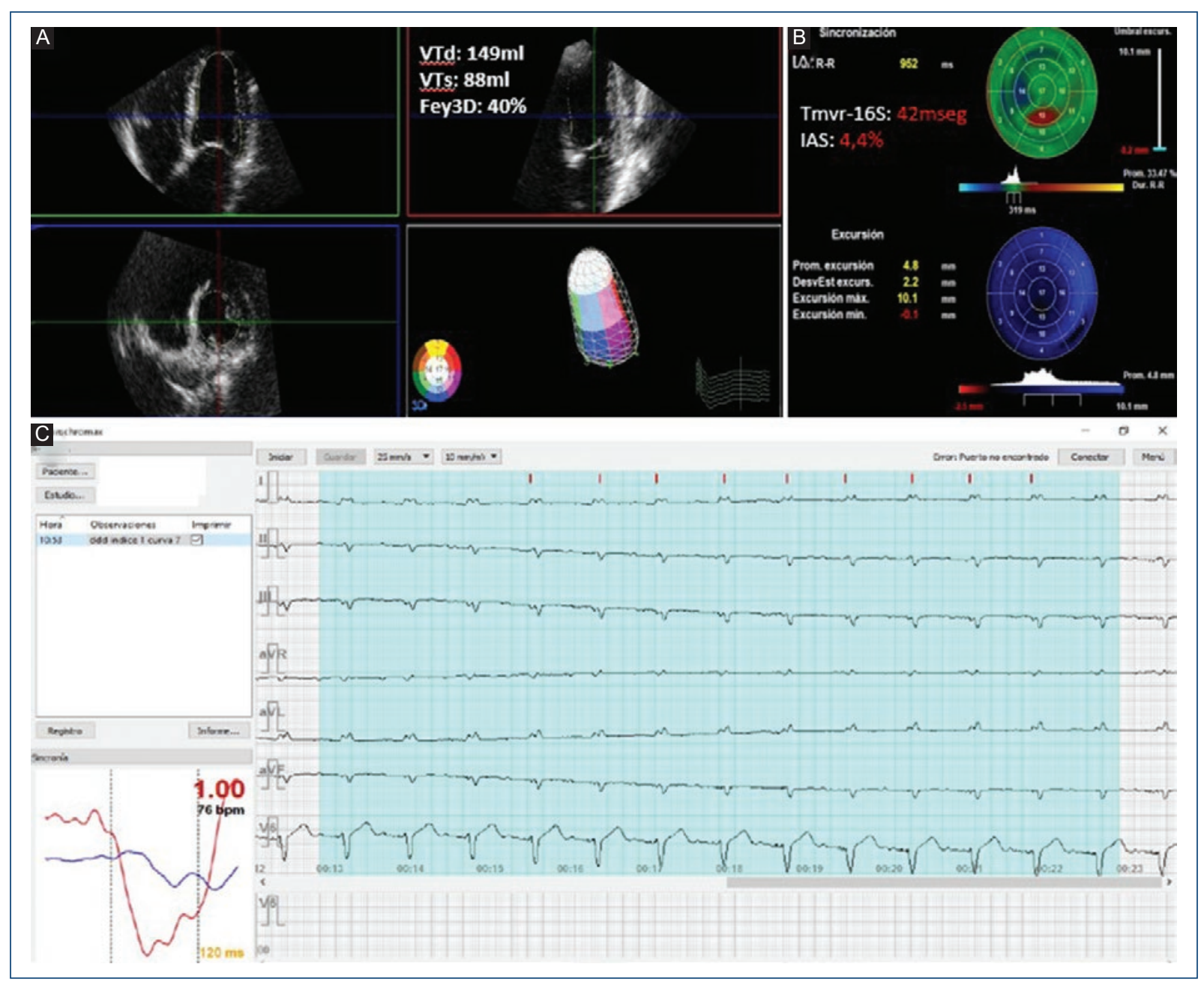

Figura 4. Estudio de la asincronía ventricular izquierda en paciente con marcapasos permanente con deterioro de la función sistólica unos meses después del implante admitido por cuadro de insuficiencia cardíaca. A: Adquisición volumétrica tridimensional del VI y cálculo de volúmenes ventriculares (volumen telediastólico: VTd de 149 ml; volumen telesistólico: VTs de $88 \mathrm{ml}$ ) y fracción de expulsión tridimensional (FEVI 3D de 40\%). B: Cálculo de la asincronía mecánica del VI mediante el índice de asincronía sistólica (IAS de 4.4\%) y la dispersión del tiempo al punto máximo de los 16 segmentos del VI (Tmvr-16S de 42 ms). C: Cálculo de la asincronía eléctrica mediante sistema no invasivo Synchromax con cálculo de un índice de asincronía de 1 (gravemente asincrónico).

técnica registraron una menor fracción de expulsión y un mayor volumen al final de la diástole en comparación con los controles normales. Se estableció además que los enfermos con hipertrofia ventricular izquierda y una tasa de estimulación mayor de $40 \%$ durante los últimos seis meses fueron parámetros relacionados con la presencia de mayor asincronía mecánica del $\mathrm{V}^{31}$.

Por lo regular, la duración del ancho del QRS ha sido el parámetro de elección para diferenciar a los pacientes que se benefician de una TRC, sin perder de vista que el aumento de la duración del QRS es una expresión de mayor asincronía eléctrica ventricular. Sin embargo, hasta la fecha no se dispone de un método cuantitativo de responder esta pregunta. En la actualidad, el nuevo sistema no invasivo Synchromax ha permitido la cuantificación del grado de asincronía ventricular en una escala de 0 a 1 en distintos escenarios clínicos, incluidos los pacientes con estimulación apical. De manera adicional, hasta la fecha no se han correlacionado estos hallazgos con los datos de la asincronía mecánica resultante de estos pacientes. La ecocardiografía ha demostrado ser útil en sus distintas modalidades para la cuantificación de este fenómeno. 
Si se considera que la resincronización es una alternativa terapéutica para mejorar la función ventricular en individuos con insuficiencia cardíaca, y que a su vez la indicación debe ser precisa para obtener el mejor resultado, es importante detectar la alteración de la sincronía mecánica. El mero hallazgo de asincronía eléctrica parece no confirmar la existencia de asincronía mecánica y es necesario obtener información de ambas alteraciones para definir conductas que hagan posible modificar las decisiones clínicas. El avance de la tecnología ofrece en la actualidad herramientas más exactas para estos estudios, como el Syncromax, para estudiar la actividad eléctrica y la ecocardiografía en sus distintas modalidades y analizar la actividad mecánica. No existen hoy día estudios que analicen la correlación existente entre el estudio de la actividad eléctrica con esa técnica y el de la mecánica con la referida al utilizar ultrasonido; se requieren otros estudios de investigación que determinen si existe o no un vínculo definido entre las alteraciones eléctricas y las mecánicas (Fig. 4).

\section{Conclusiones}

El estudio de la asincronía eléctrica y mecánica como resultado de la estimulación apical permanente del VD puede ocasionar deterioro de la función sistólica del VI y la aparición de insuficiencia cardíaca. La detección y estudio oportunos de esta alteración son cruciales para identificar a los pacientes que se benefician de una transición a un tratamiento de resincronización cardíaca. El cálculo de la asincronía eléctrica resultante de la cuantificación mediante el sistema Synchromax y la asincronía mecánica como consecuencia de ésta estudiada con la ecocardiografía en sus distintas modalidades son técnicas que facilitarían tomar decisiones clínicas. A pesar de ello, se requieren estudios de cohorte de pacientes que posibiliten establecer con precisión la presencia de ambos fenómenos y a la vez determinar los puntos de corte a partir de lo cual un grado de asincronía eléctrica produzca asincronía mecánica del VI.

\section{Financiamiento}

La presente investigación no ha recibido ninguna beca específica de agencias de los sectores público, comercial, o sin ánimo de lucro.

\section{Conflicto de intereses}

Ninguno.

\section{Responsabilidades éticas}

Protección de personas y animales. Los autores declaran que para esta investigación no se han realizado experimentos en seres humanos ni en animales.

Confidencialidad de los datos. Los autores declaran que en este artículo no aparecen datos de pacientes.

Derecho a la privacidad y consentimiento informado. Los autores han obtenido el consentimiento informado de los pacientes o sujetos referidos en el artículo. Este documento obra en poder del autor de correspondencia.

\section{Bibliografía}

1. Tops LF, Schalij MJ, Bax JJ. The effects of right ventricular apical pacing on ventricular function and dyssynchrony implications for therapy. J Am Coll Cardiol. 2009;54:764-776.

2. Sweeney MO, Hellkamp AS, Ellenbogen KA, Greenspon AJ, Freedman RA, Lee KL, et al. Adverse effect of ventricular pacing on heart failure and atrial fibrillation among patients with normal baseline QRS duration in a clinical trial of pacemaker therapy for sinus node dysfunction. Circulation. 2003;107:2932-2937.

3. Vassallo JA, Cassidy DM, Miller JM, Buxton AE, Marchlinski FE, Josephson ME. Left ventricular endocardial activation during right ventricular pacing: effect of underlying heart disease. J Am Coll Cardiol. 1986;7:1228-1233.

4. Prinzen FW, Hunter WC, Wyman BT, McVeigh ER. Mapping of regional myocardial strain and work during ventricular pacing: experimental study using magnetic resonance imaging tagging. J Am Coll Cardiol. 1999;33:1735-1742.

5. Nahlawi M, Waligora M, Spies SM, Bonow RO, Kadish AH, Goldberger JJ. Left ventricular function during and after right ventricular pacing. J Am Coll Cardiol. 2004;44:1883-1888.

6. Tayal B, Fruelund P, Sogaard P, Riahi S, Polcwiartek C, Atwater BD et al. Incidence of heart failure after pacemaker implantation: a nationwide Danish Registry-based follow-up study. European Heart Journal. 2019;40(44):3641-3648, https://doi.org/10.1093/eurheartj/ehz584.

7. Antoni ML, Boden H, Hoogslag GE, Ewe SH, Auger D, Holman ER, et al. Prevalence of dyssynchrony and relation with long-term outcome in patients after acute myocardial infarction. Am J Cardiol. 2011;108:16891696.

8. Vassallo JA, Cassidy DM, Miller JM, Buxton AE, Marchlinski FE, Josephson ME. Left ventricular endocardial activation during right ventricular pacing: effect of underlying heart disease. J Am Coll Cardiol. 1986;7:12281233.

9. Lin LY, Wu CK, Juang JM, Wang YC, Su MY, Lai LP, et al. Myocardial regional interstitial fibrosis is associated with left intraventricular dyssynchrony in patients with heart failure: a cardiovascular magnetic resonance study. Sci Rep. 2016;6:20711.

10. Curtis $A B$. Biventricular pacing for atrioventricular block and systolic dysfunction. N Engl J Med. 2013;369:579.

11. Ponikowski P, Voors AA, Anker SD, Bueno H, Cleland JG, Coats AJ, et al. ESC Scientific Document Group. 2016 ESC Guidelines for the diagnosis and treatment of acute and chronic heart failure: the Task Force for the diagnosis and treatment of acute and chronic heart failure of the European Society of Cardiology (ESC). Developed with the special contribution of the Heart Failure Association (HFA) of the ESC. Eur Heart J. 2016:37:2129-2200.

12. Yu CM, Chan JY, Zhang Q, Omar R, Yip GW, Hussin A, et al. Biventricular pacing in patients with bradycardia and normal ejection fraction. N Engl J Med. 2009;361:2123-2134.

13. Kiehl EL, Makki T, Kumar R, Gumber D, Kwon DH, Rickard JW, et al. Incidence and predictors of right ventricular pacing induced cardiomyopathy in patients with complete atrioventricular block and preserved left ventricular systolic function. Heart Rhythm. 2016;13(12):2272-2278, doi: 10.1016/j.hrthm.2016.09.027.

14. Fang F, Yat-Sun Chan J, Wai-Kwok Yip G, Xie J, Zhang Q, Wing-Hong Fung $\mathrm{J}$, et al. Prevalence and determinants of left ventricular systolic dyssynchrony in patients with normal ejection fraction received right ventricular apical pacing: a real-time three-dimensional echocardiographic study. European Journal of Echocardiography. 2010;11:109-118.

15. Wood PW, Choy JB, Nanda NC, Becher H. Left ventricular ejection fraction and volumes: it depends on the imaging method. Echocardiography. 2014;31(1):87-100. 
16. Driessen MM, Kort E, Cramer MJ. Assessment of LV ejection fraction using real-time $3 \mathrm{D}$ echocardiography in daily practice: direct comparison of the volumetric and speckle tracking methodologies to CMR. Neth Heart J. 2014;22(9):383-90.

17. Hoffmann R, Barletta G, Bardeleben S. Analysis of left ventricular volumes and function: a multicenter comparison of cardiac magnetic resonance imaging, cine ventriculography, and unenhanced and contrast-enhanced two-dimensional and three-dimensional echocardiography. J Am Soc Echocardiogr. 2014;27(3):292-301.

18. Chung ES, Katra RP, Ghio S. Cardiac resynchronization therapy may benefit patients with left ventricular ejection fraction $>35 \%$ : a PROSPECT trial substudy. Eur J Heart Fail. 2010;12(6):581-7.

19. Everdingen WM, Schipper JC, van't Sant J, Ramdat Misier K, Meine M, Cramer MJ. Echocardiography and cardiac resynchronisation therapy, friends or foes?. Netherlands Heart Journal. 2015;24,10.1007/s12471015-0769-3.

20. Zuloaga C. Qué aprendimos de sincronía biventricular con el uso de "Syncromax". Rev Electro y Arritmias. 2015;9:38-43.

21. Perry R, De Pasquale CG, Chew DP. QRS duration alone misses cardiac dyssynchrony in a substantial proportion of patients with chronic heart failure. J Am Soc Echocardiogr. 2006;19:1257-1263.

22. Bonomini MP, Ortega DF, Barja LD, Logarzo E, Mangani N, Paolucci A. ECG parameters to predict left ventricular electrical delay. Journal of Electrocardiology. 2018;51(5):844-850.

23. Bonomini MP, Ortega DF, Barja LD, Mangani N, Paolucci A. Electrical approach to improve left ventricular activation during right ventricle stimulation. Medicina (Buenos Aires). 2017;77(1):7-12.

24. Villarroel-Ábrego $\mathrm{H}$, Garillo R. Estimulación del ventrículo derecho como causante y agravante de disincronía ventricular. Rev. Costarricense de Cardiología. 2018;20(2).
25. Yu CM, Zhang Q, Fung JW, Chan HC, Chan YS, Yip GW, et al. A novel tool to assess systolic asynchrony and identify responders of cardiac resynchronization therapy by tissue synchronization imaging. J Am Coll Cardiol. 2005;45:677-84.

26. Wang $\mathrm{H}$, Shuraih $\mathrm{M}$, Ahmad M. Real time three-dimensional echocardiography in assessment of left ventricular dyssynchrony and cardiac resynchronization therapy. Echocardiography. 2012;29:192-9.

27. Macron L, Lim P, Bensaid A, Nahum J, Dussault C, Mitchell-Heggs L, et al. Single-beat versus multibeat real-time 3D echocardiography for assessing left ventricular volumes and ejection fraction: a comparison study with cardiac magnetic resonance, Circ Cardiovasc Imaging. 2010;3:450-5

28. Wang YC, Yu CC, Chiu FC, Klepfer R, Hilpisch K, Splett V, et al. Impact of ventricular dyssynchrony on postexercise accommodation of systolic myocardial motion in hypertensive patients with heart failure and a normal ejection fraction: a tissue-Doppler echocardiography study. J Card Fail. 2012;18:134-9).

29. Delgado V, Sitges M, Vidal B, Silva E, Azqueta M, Tolosana JM, et al Assessment of left ventricular dyssynchrony by real-time three-dimensional echocardiography. Rev Esp Cardiol. 2008;61:825-34).

30. Liu WH, Chen MC, Chen YL. Right ventricular apical pacing acutely impairs left ventricular function and induces mechanical dyssynchrony in patients with sick sinus syndrome: A real-time three-dimensional echocardiographic study. J Am Soc Echocardiogr. 2008;21(3):224-229.

31. Fang F, Yat-Sun Chan J, Wai-Kwok Yip G, Xie J, Zhang Q, Wing-Hong Fung J, et al. Prevalence and determinants of left ventricular systolic dyssynchrony in patients with normal ejection fraction received right ventricular apical pacing: a real-time three-dimensional echocardiographic study. European Journal of Echocardiography. 2010;11: 109-118. 